\title{
Nonlinear conductance in molecular devices: molecular length dependence
}

\author{
Ž. Crljer * \\ R. Bošković Institute, P.O. Box 180, 10002 Zagreb, Croatia \\ A. Grigoriev and G. Wendin \\ Department of Microtecnology and Nanoscience, MC2, \\ Chalmers University of Technology, SE-41296, Göteborg, Sweden \\ K. Stokbro \\ Nanoscience Center, Copenhagen University, Universitetsparken 5d DK-2100 Copenhagen east, Denmark
}

(Dated: August 12, 2004)

\begin{abstract}
We theoretically study the electronic transport in the monolayer of dithiolated phenylene vinylene oligomeres coupled to the (111) surfaces of gold electrodes. We use non-equilibrium Green functions (NEGF) and density functional theory(DFT) implemented in the TranSIESTA package to obtain a full ab initio self-consistent description of the transport current through the molecular nanostructure with different electrochemical bias potentials. The calculated current-voltage characteristics (IVC) of the systems for the same contact geometry have shown a systematic decrease of the conductivity with the increased length of the molecules. We analyze the results in terms of transmission eigenchannels and find that besides the delocalization of molecular orbitals the distance between gold electrodes also determines the transport properties.
\end{abstract}

PACS numbers: 85.65.+h; 73.63.-b; 71.15.Mb

Keywords: Nonequilibrium electron transport: Density functional calculations; Organic molecules; Nanostructure

\section{INTRODUCTION}

Since the pioneering work on electron transport through molecules by Aviram and Ratner ${ }^{\underline{1}}$ there has been remarkable progress in the field of molecular electronics. With the advances of experimental tools and techniques, various molecular devices have shown a number of interesting effects: negative differential resistance, $2, \frac{2}{,}$ switching properties, ${ }^{3}$ memory effects. $\stackrel{4}{-}$ A number of measurements have been made where the electrical current has been driven through monolayer molecular films between metallic electrodes, including break junction,,$\frac{5}{,}$ and evaporated electrode ${ }^{6}$ experiments. The connection of a single molecule to conducting electrodes has been achieved using $\mathrm{STM}^{7}$ and break junction ${ }^{6.8}$ techniques. Owing to their stability the most common systems considered were thiol-ended organic molecules on gold electrodes.

The current-voltage measurement by Reed et al $\underline{\underline{6}}$ of dithiol benzene molecule (dtb) onto gold electrodes in break-junction experiments showed an apparent gap of about $0.7 \mathrm{~V}$. It raised the question of its nature: Whether it was a Coulomb gap or it reflected the mismatch between the contact Fermi level and the lowest unoccupied molecular orbital. It was viewed as a question of bonding between the molecule and the electrodes.

In general, in the nonbonding, and even weak-bonding situations one expects no coherent transport, but instead a sequential charging of the molecule and Coulomb blockade effects. Recently Kubatkin et al ${ }^{\underline{9}}$ have demonstrated a Coulomb blockade and different charge states in the junction with weakly coupled (physisorbed) OPV5 molecule onto gold contacts.
In the strong-bonding case, a coherent transport is dominant and the staircaselike structure could appear owing to the succession of scattering resonances entering the bias window ${ }^{5}$ In many junctions no apparent gap was noticed. Instead, linear current-voltage curves were reported at low bias for strongly bonded thiolate molecules. There are a number of possible transport mechanisms that give rise to linear IVC. In the evaporation electrode technique, for example, grainy surfaces can form with the likely protrusion of electrode atoms in between the molecules of the monolayer, thus spoiling the surface order and the dominant conduction mechanism appears to be hopping with the characteristic energy scale of 10-100 $\mathrm{meV}, 10$

In order to explain experimental IVCs, the dithiolbenzene molecule coupled to $\mathrm{Au}(111)$ surfaces was a kind of model system for theoretical studies. After the first semiempirical investigations,, 11.12 ab initio calculations within the framework of density functional theory were performed. The conductance was found to be more than an order of magnitude larger than in experiment, 13,14 despite the assumption of unequal bonding of the molecule to the electrode surfaces ${ }^{15}$

In the theoretical model of molecular junction with parallel perfect electrode surfaces, it came natural that the electronic structure of the system provided a finite density of states at the Fermi level of the coupled system, ruling out the appearance of the gap in the I-V curves. The theoretically obtained conductances were as a rule more than an order of magnitude larger compared with the measured ones. The difference was related to the varying strengths of the sulfurgold bonds and to the adsorption sites. Interestingly, 
for longer molecules, quantitative agreement of theoretical considerations 16.17 .18 is considerably improved when compared with the measurements 8.19

The transmission properties of the systems are determined by the electronic structure of the combined molecule and electrode systems. The distance between the electrodes has an important role in the conduction of the junction. In simple terms, the metal-molecule-metal system can be viewed as a finite quantum well that sets up in between the metal surfaces. The direct tunneling between the electrodes, despite the possible electrodeinduced gap states in the metal-air-metal junctions, is considered to be too small to contribute considerably to the current of the typical size molecular junctions $\frac{5}{5}$

With the molecule bridging the interelectrode gap, a possibility for the molecule-induced interelectrode gap states, or resonances appears. Those states will have an important impact on the transport properties. The induced gap states, being molecule mediated, depend on the length of the molecule, and on the interelectrode separation. The transport is determined by scattering states which are Bloch-like states in the electrodes and molecular orbitals like states in between the electrodes.

Previous studies of the electronic transport of the junctions with the molecules of different length included a number of oligomeres in a search for a good molecular wire ${ }^{20.21}$ For this purpose, one has to consider the molecules with the same basic unit that repeats successively with the length of the molecule. The electronic structure at the surface and the adsorption site geometry for that reason should be similar for all the adsorbed molecules considered. Magoga and Joachim ${ }^{22}$ used a semi-empirical approach in their studies of the tunnel transport regime of certain families of oligomeres. They considered the transmission at zero bias voltage and found that the conductance follows a simple exponential decrease with the length of the molecule. However, in order to get a full account of the details of the electronic structure of the electrodes, the electrode surfaces, the molecule, and the molecule-induced gap states on the transport properties of the junction a full ab initio approach is needed. Moreover, as the junction is subject to the non-equilibrium conditions the transmission should be obtained self-consistently for each bias voltage applied across the junction.

In this paper, we therefore use the non-equilibrium Green functions technique and the density functional theory to obtain a full ab initio self-consistent description of the transport properties. We investigate the conductance of the sulfur-ended phenylene vynelene oligomeres coupled to gold electrodes; OPVn systems, with n equal to the number of benzene rings in the molecule. The number of benzene rings of these oligomeres determines the length of the molecule. Owing to a strong bonding of sulfur on metal surfaces, particularly gold, all other parameters of the system can be kept fixed for the systems with different molecules of the series. The difference in the transport properties of the coupled systems then re- flects the electrode distance in addition to the electronic structure of the molecule. The results will show two different transport regimes ${ }^{23}$; the one where the electrodes are close enough that the induced gap states give an appreciable contribution to the current, and the other where the separation between the electrodes is large enough to result in their negligible contribution.

In Sec. III of the paper we first report on the model used and the generalities of the computational procedure. In Sec. IIII we calculate the current through the system as a function of applied bias voltages and analyze them in terms of the evolution of transmission spectra. We discuss the results in Sec. IV

\section{CALCULATIONS}

A typical molecular electronic system consists of a molecule coupled to two electrodes with different electrochemical potentials. Depending on the experimental method of building, the device may vary the contact geometry with respect to the adsorption site as well as to the adsorption strength ${ }^{24}$ We consider an idealized geometry with a molecule symmetrically coupled to the two identical gold electrodes.

\section{A. Computational method}

To perform the first-principle quantum modeling of the electronic structure under non-equilibrium conditions and to calculate the current-voltage (I-V) characteristics of the system, we used non-equilibrium Green functions technique based on density functional theory as implemented in the recently developed TranSIESTA simulation package ${ }^{25}$ Core electrons were modeled with Troullier-Martins ${ }^{26}$ soft norm-conserving pseudopotentials and the valence electrons were expanded in a SIESTA localized basis set 27 The system was divided into three regions: the left and right electrodes and the central region. The central part contained the portion of physical electrodes, where all screening effects took place. The electronic occupations of the system was determined by the electrochemical potentials of the electrodes. The charge distribution in the electrodes corresponded to the bulk phases of the same material.

The density matrix of the system under the external bias was calculated self-consistently within density functional theory (DFT) using the only major approximation of the method in the choice of the exchange correlation functional. We used the local density approximation (LDA). The virtue of the method is that it gives the Hamiltonian in the same form as in the empirical tight-binding approach making its techniques straightforwardly applicable. The nonlinear current through the 


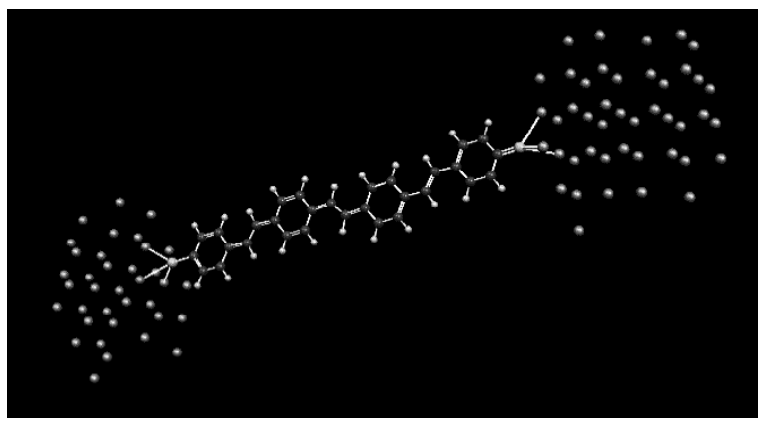

FIG. 1: A phenylene vinylene oligomere, OPV4, connected to two $\mathrm{Au}(111)$ surfaces via thiolate bonds.

contact is calculated using the Landauer formula 28

$$
I\left(V_{b}\right)=G_{0} \int_{\mu_{L}}^{\mu_{R}} T\left(E, V_{b}\right) d E
$$

where $G_{0}=2 e^{2} / h$ is the quantum unit of conductance and $\mu_{L / R}$ are electrochemical potentials of the left and right electrodes. With the applied bias potential $e V_{b}$, the left and right potentials become $\mu_{L}\left(V_{b}\right)=\mu_{L}(0)+e V_{b} / 2$ and $\mu_{R}\left(V_{b}\right)=\mu_{R}(0)-e V_{b} / 2$, respectively. The energy region of the transmission spectrum that contributes to the current integral in Eq.1. we refer to as the bias window. The total transmission probability $T\left(E, V_{b}\right)=$ $\sum_{n=1}^{N} T_{n}\left(E, V_{b}\right)$ for electrons incident at an energy $\mathrm{E}$ through the device under the potential bias $V_{b}$ is composed of all available conduction channels with the individual transmission $T_{n}$.

\section{B. OPVn molecules bonded to gold electrodes}

The aim of this paper is to investigate the electrical and structural properties of the monolayer of phenylene vinylene oligomere molecules (OPVn) coupled to gold electrodes via the sulfur atoms on both ends. There are good reasons to believe that the result for the monolayer of OPVn molecules applies to the single molecule, too 29.30

It is usually assumed that sulfur forms thiolate bonds with the gold surface, which is not necessarily always the case. Depending on the absorption technique, if the strong SH bond is not cleaved and if the molecule forms a thiol bond with gold, a preferential adsorption site might be at the top with the nonzero tilt angle 31 In our calculation, however, we consider thiolate bonds at a 3 -fold hollow site and the tilt angle of the molecular axis to the surface normal to be zero. We have also made the calculation with molecule adsorbed in the on-top position on the gold atom at both electrodes, and found that the adsorption at the 3 -fold hollow site is energetically favorable to the on-top absorption by $2.6 \mathrm{eV}$. In the experimental situation, some molecules in the self-assembled monolayer will be atypical bonded to various kinds of defects on the surface. We, however, consider an idealized situation and assume further that all molecules align with parallel phenyl rings. There is a good reason to believe that this is energetically more favorable, since it gives a larger $\pi$ overlap.

The geometry of the system was determined as follows. We used a (3x3) unit cell of the $\mathrm{Au}(111)$ surface for the electrodes and assumed that the molecule of cis conformation was chemisorbed to the surface of the electrodes, as shown in Fig प The OPV3 molecule was positioned symmetrically in the hollow site of both $\mathrm{Au}(111)$ surfaces. After positioning the molecule perpendicularly in the z-direction so that it has a favorable $\mathrm{Au}-\mathrm{S}$ bonding distance,$\stackrel{32}{ }$ we let its molecular coordinate to relax until the average force on the molecular atom was less than $0.04 \mathrm{eV} / \AA$. The gold atoms were kept fixed at their bulk positions.

The same procedure was followed for OPV4 and OPV5 molecules. The obtained distances for $\mathrm{Au}-\mathrm{S}$ atoms were $2.39 \AA$ for all systems considered within the computational accuracy, the convergence in total energy tolerance was $0.0001 \mathrm{eV}$ and the force tolerance was $0.04 \mathrm{eV} / \AA$. In order to get an additional check on the stability of the results with respect to the $\mathrm{Au}-\mathrm{S}$ distance, we made a calculation for the OPV3 system with an Au-S distance enlarged by $0.1 \AA$. The minor effects on the transmission with no consequences on the current through the system were obtained. The lack of the force dependence on the transport properties of the system with chemically bonded contacts was observed by Cui et al ${ }^{19}$ on octaneditiol molecules.

In the TranSIESTA procedure the charge on the molecule is not fixed. It adjusts itself to minimize the free energy as the electrochemical potentials of the electrodes are changed. For the systems considered, we have found that molecules preserve charge neutrality to within 0.15 e with the change of the bias voltage.

The important aspect of the calculation is the robustness of the results to computational details. From the calculation by Stokbro et al. ${ }^{25}$ for the dithiolate benzene molecule on $\mathrm{Au}(111)$ surfaces (dtb) in the hollow position we know that the use of the generalized gradient approximation (GGA) instead of the LDA gives almost negligible effects on the transmission spectra. The unimportance of the relaxation of the first two gold layers for final results was also demonstrated. As the binding geometry is mostly determined in the first two layers of gold atoms, the conclusion is valid for the systems with OPV3, OPV4 and OPV5 molecules as well. 

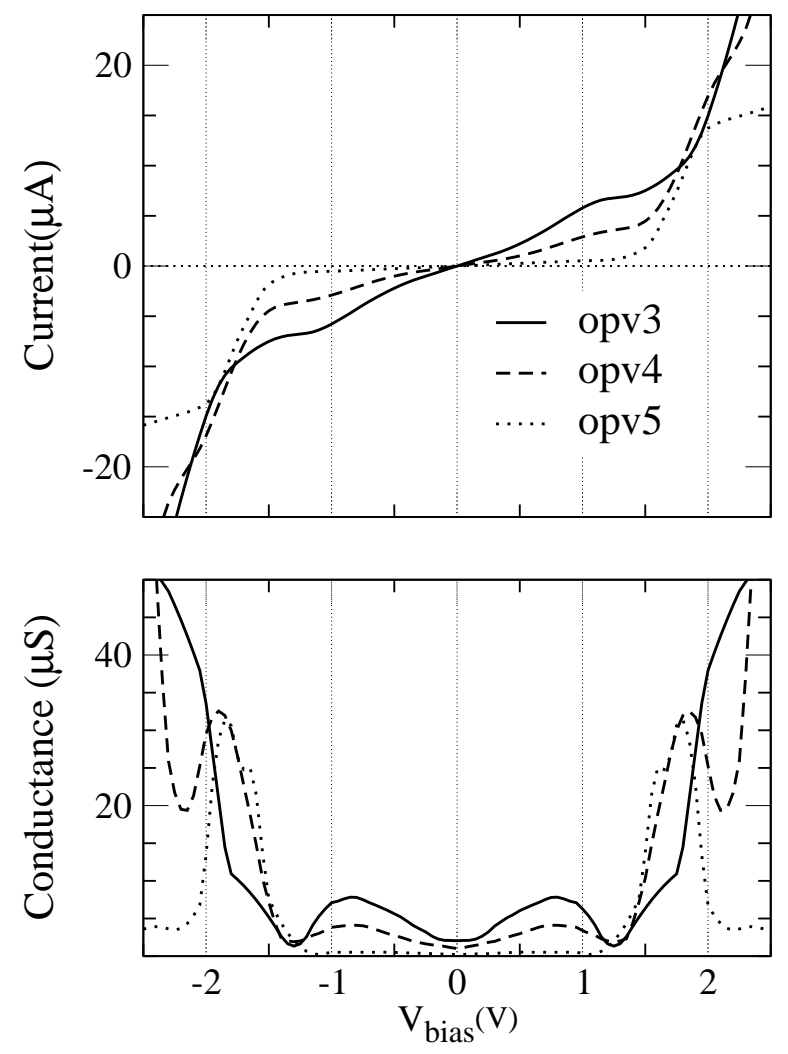

FIG. 2: Current and differential conductance of OPVn systems as a function of bias. A self-consistent calculation has been employed for each bias voltage.

\section{TRANSMISSION PROPERTIES AND I-V CHARACTERISTICS}

In order to obtain I-V spectra and differential conductance, we performed self-consistent calculations for a bias voltage in the range from $-3.0 \mathrm{~V}$ to $3.0 \mathrm{~V}$. Since our electronic structure in the contact region is fully symmetric, the corresponding I-V spectra are symmetric with respect to the reversed bias.

The general trend of $\mathrm{I}-\mathrm{V}$ curves is shown in Fig 2. One observes that all curves have a similar general shape. They show a slow increase of the current with the bias voltage up to a certain bias and then a steep increase at a point where transmission resonances come into alignment with the bias window.

Two characteric features of the systems can be observed. The first one is that the current in the ohmic region of the transport is lower as the length of the molecule is larger. The second one is that a steep increase of the current of the longer molecule happens at smaller bias than for the short molecule.

When compared to the results for shorter DTB system $^{15}$ the absolute value of the current of the OPV3 is almost an order of magnitude smaller. It is also in good agreement with the current of the similar type of molecules. 8,17
The zero bias conductance $G=\frac{e^{2}}{h} T\left(\mu_{L / R}, 0\right)$ is also much smaller than for DTB. They are 2.1 $\mu S$ for OPV3, $1.0 \mu S$ for OPV4, $0.2 \mu S$ for OPV5, in comparison with $35.0 \mu S$ for DTB.

In the following we analyze the obtained IVCs for OPV3-5 in terms of the dependence of the transition spectra on molecular length (number of benzene rings), bias voltage, and interelectrode separation.

\section{A. OPV3}

Chemisorption of the molecule on the gold surfaces leads to the change of the energy levels in both systems. In particular, some of the molecular levels broaden into a continuum. The eigenstates of the combined system become the scattering states which are Bloch-wavelike in the electrode and molecular orbital-like in the molecule. 33 The more delocalized the state is, more spreading the corresponding transmission peak shows.

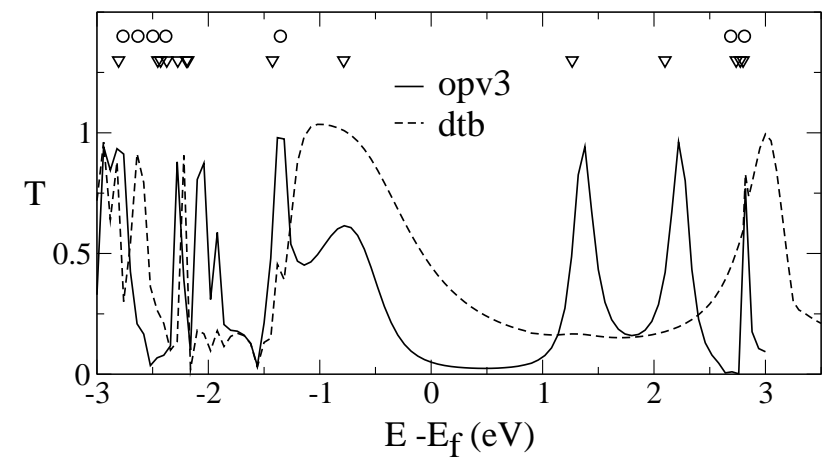

FIG. 3: Transmission amplitude of DTB and OPV3 molecules on $\mathrm{Au}(111)$ surfaces at zero bias voltage. MPSH eigenvalues are marked with circles for dtb and with triangles for OPV3.

In Fig 3 we compare the transmission spectra of the coupled dtb system with the OPV3 system at zero bias. In the figure we also mark the eigenvalues of the projected self-consistent Hamiltonian (MPSH) onto the molecular orbitals. The transmission spectra are proportional to the density of states of the combined system. The MPSH levels are normally broadened and shifted in energy by the interaction with the gold surface. Some of the states have a large weight on the $\mathrm{S}$ atom and form a strong bond with the gold electrode. The others may have a little overlap with Au states and form a narrow peak in the density of states and in the transmission spectra.

In the DTB system the huge transmission resonance below the Fermi level is due to a superposition of strongly hybridized levels, as demonstrated in Ref. 15.

The OPV3 system does not exhibit such a huge resonance as DTB does and it has a considerably less spectral weight at the Fermi level. The OPV3 resonance below the Fermi level is built on the HOMO molecular state at the energy $E=-0.799 \mathrm{eV}$. The reason for being less 

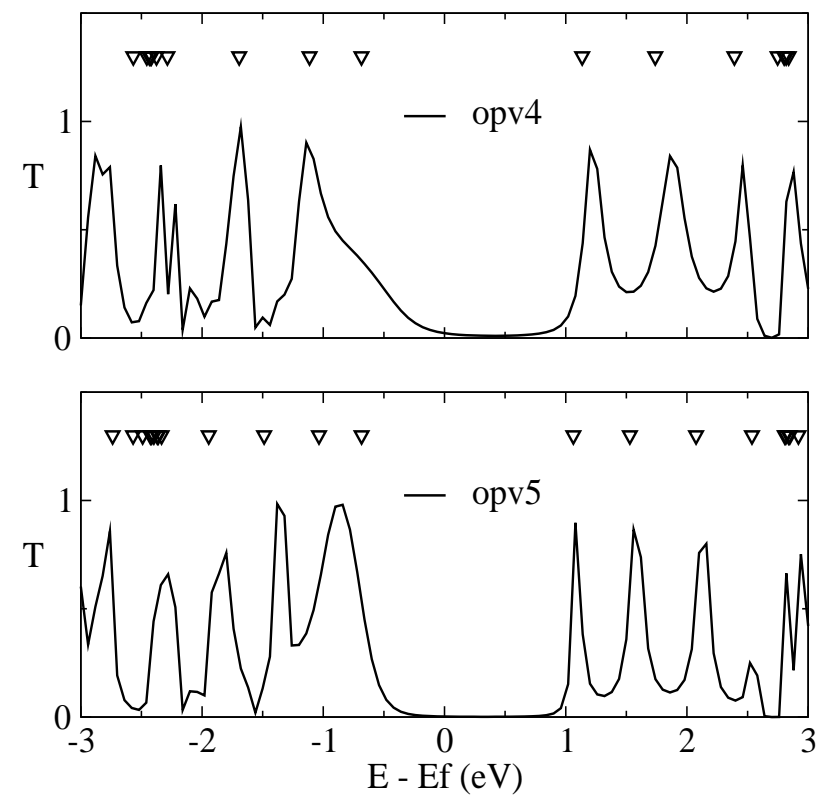

FIG. 4: Transmission amplitude and MPSH eigenvalues of OPV4 and OPV5 systems at zero bias.

pronounced than in DTB is the larger separation of Au electrodes in OPV3, resulting in the smaller overall overlap of gold states with the molecular states. This leads to smaller hybridization and less broadened levels resulting in lower density of states around the Fermi level.

When the bias $V_{b}$ is applied to the system the chemical potential of the electrodes changes. The calculated transmission spectra are plotted for a set of bias values in Fig [5 The main effect of the increased bias is that more of the resonance gets into the bias window. The position of the HOMO related transmission resonance does not change with respect to the average electrochemical potential as the bias is increased, but its weigth diminishes. The resonance gives a lower contribution to the current tranport at higher bias, even when the maximum of the resonance enters the bias window. That peculiar bias dependence of the HOMO resonance leads to a step-like behavior of the current and a dip in the conductance at the bias of $1.25 \mathrm{~V}$ as seen in Fig 2

The transmission peaks corresponding to LUMO, and higher levels change linearly with the applied bias, contrary to the HOMO peak. They are slowly approaching the electrochemical potential of the left electrode leading to a smaller HOMO-LUMO separation. The net result is the lower transmission in the HOMO-LUMO region. The current is considerably smaller when compared to the current of the smaller dtb system, particularly at low bias. Eventually, at high enough bias, at above approximately $2 \mathrm{~V}$ the LUMO resonance enters significantly the bias window and contributes dominantly to the current. This is the region of the steep increase of the current as seen in Fig 2] Nevertheless the current in the OPV3 system is almost an order of magnitude smaller than in the DTB system in the region we considered.

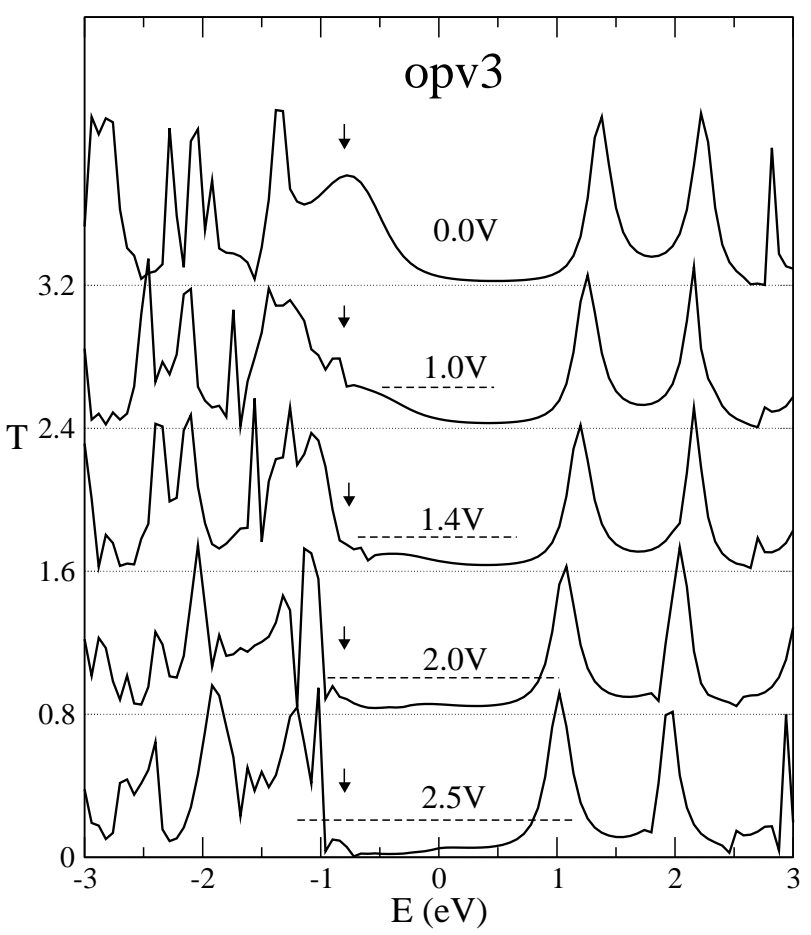

FIG. 5: Transmission $T\left(E, V_{b}\right)$ of the OPV3 system as a function of the bias voltage (shifted vertically by 0.8 for visibility). Energies are relative to the average electrochemical potential $\left(\mu_{L}+\mu_{R}\right) / 2$. The bias windows are marked with dashed lines. The amplitude corresponding to the HOMO levels (marked with arrows) decrease with the increased bias.

\section{B. OPV4}

There is a clear separation of HOMO and LUMO levels in the OPV4 system for zero bias, as shown in Fig 4 The appearance of the lower transmission of the HOMO related resonance and the lower density of states at $E_{f}$ is more pronounced than in OPV3 system. The position of the HOMO level is $0.685 \mathrm{eV}$ below the Fermi level. The LUMO level is $1.136 \mathrm{eV}$ above the Fermi level and is less broaden giving the lower tail at the Fermi level. The behavior is maintained at the finite bias, as seen in Fig [6] The MPSH levels are narrower in general, and the resulting transmission at low bias is smaller when compared with the OPV3 system as seen in Fig 2 Like in the OPV3 a dip in the conductance appears at $1.25 \mathrm{~V}$.

\section{OPV5}

The tendency of the appearance of a sharper welldefined peaks in the system with the longer molecule is clearly demonstrated in the OPV5 system in Fig 4 The contribution of the underlying states to the resonance is obviously changed with respect to the OPV4. The overall overlap of the molecular states with the gold electrode states is lower leading to the narrowing of the 


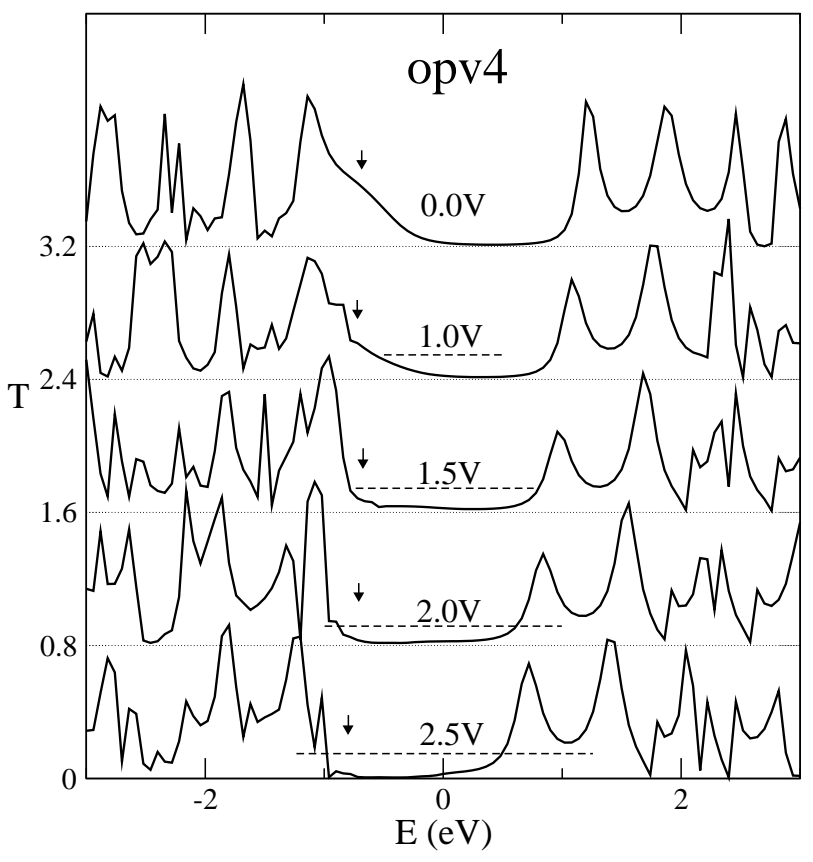

FIG. 6: Transmission $T\left(E, V_{b}\right)$ of the OPV4 system for different bias voltages The notation is the same as in Fig 5 The HOMO amplitude decreases with increased bias and the LUMO amplitude moves towards the average electrochemical potential of the electrodes.

transmission resonances. The HOMO and LUMO resonances at zero bias are at $0.684 \mathrm{eV}$ below and $1.063 \mathrm{eV}$ above the Fermi level, respectively. With the increased bias, the HOMO transmission almost disappears, while the LUMO slightly moves towards the average chemical potential of the electrodes. The dip in the conductance at $1.25 \mathrm{~V}$, which was present in OPV3 and OPV4, is missing in OPV5 owing to a very little spectral weigth of the HOMO resonance.

There is a new feature in the spectra related to the narrowing of the levels. An almost pure HOMO-LUMO gap sets up at any bias. The bias dependence of the gap is reflected only through its slight shift with respect to the average chemical potential of the electrodes, as seen in Fig 7 The low zero-bias conductance is $0.2 \mu S$, and the current is distinctly lower compared with the preceding member of the OPVn series.

\section{DISCUSSION}

The current-voltage dependence of the systems can be obviously understood in terms of the transmission resonances. The OPV3 system at zero bias does not show a huge transmission resonance below the Fermi level, as was characteristic of a smaller DTB system. The tendency of diminishing transmission and density of state around the Fermi level is more pronounced for the OPV4 system and even more for the OPV5. Loosely

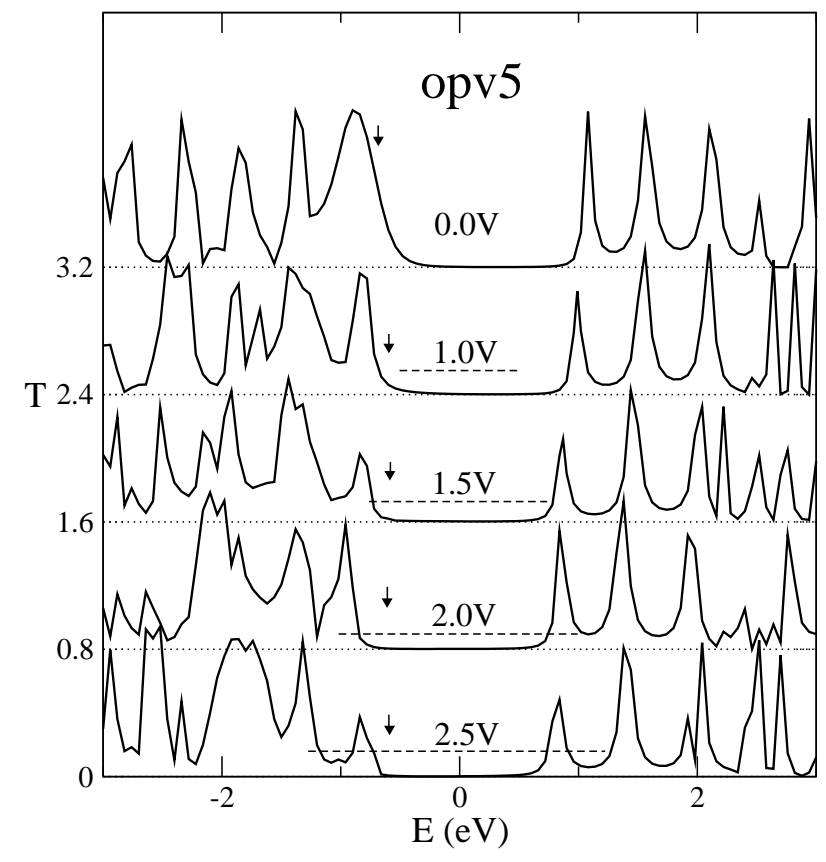

FIG. 7: Transmission $T\left(E, V_{b}\right)$ of the OPV5 system. The notation is the same as in Fig[5] The behavior of the HOMO and LUMO amplitudes is similar to that of the OPV3 and OPV4 systems. Note a low density in the HOMO-LUMO region.

speaking, for a short molecule there will be a strong molecule-mediated overlap of electrode states leading to wide transmission resonances as opposed to the weaker overlap, and more narrow transmission peaks for longer molecules.

To understand this consideration better, let us consider the transmission related to the interelectrode separation. We define it as the distance between the surface planes of the left and right electrodes. In our calculations the shortest distance is in the DTB system with the value $1.05 \mathrm{~nm}$. Our input radius of the gold atoms is $3 \AA$, and the direct overlap of the gold atom states from the left and the right electrodes cannot take place. The pure gold levels, which perfectly follow the electrode chemical potential, can contribute directly to the tunneling process through the electrode-induced resonant states or even quantum well states related to gold surface states 34 The properties of these states are dependent on the separation between the electrodes. As reported by Kergueris et al.,$\frac{5}{5}$ the tunneling conductance in the goldair-gold junction is linear and of the order $0.02 \mu S$ at the barrier height of $1 \mathrm{~V}$ for the interelectrode spacing of $1 \mathrm{~nm}$. That appears to be several orders of magnitude smaller than the typical conductance of metalmolecule-metal junctions. However, with the molecule chemisorbed in between the electrodes, the electrode induced gap states and resonances play an important role in transport properties. They are, of course, a part of the hybridized molecule-electrode states, caused by the 
TABLE I: Interelectrode separation and zero-bias conductance of OPVn systems. $d_{\text {elec }}$. is the distance between the surface (111) gold planes of the left and right electrodes. $\mathrm{G}\left(\mu_{L / R}\right.$, $0)$ is the conductance at the Fermi level for zero bias.

\begin{tabular}{ccccc}
\hline \hline & DTB & OPV3 & OPV4 & OPV5 \\
\hline$d_{\text {elec. }}$ & $1.05 \mathrm{~nm}$ & $2.3 \mathrm{~nm}$ & $2.97 \mathrm{~nm}$ & $3.64 \mathrm{~nm}$ \\
$\mathrm{G}\left(\mu_{L / R}, 0\right)$ & $35 \mu S$ & $2.1 \mu S$ & $1.0 \mu S$ & $0.2 \mu S$ \\
\hline \hline
\end{tabular}

molecule-mediated overlap of electrode states. We can tentatively call them molecule-induced gap states.

If the electrodes are close, they contribute to the finite density of states and to the enhanced transmission in the HOMO-LUMO region of the combined systems. As the electrode separation is larger, the molecule-induced gap states loose the weight, and their contribution to the conducting channels of the system becomes smaller. The transmission peaks become narrower and the transmission in the HOMO-LUMO region consequently smaller.

The DTB molecule is the shortest one we have considered. The increase of the interelectrode separation in the OPVn series from $2.3 \mathrm{~nm}$ for OPV3 to $3.64 \mathrm{~nm}$ for OPV5 is followed by the decrease of the zero-bias conductance, as seen in Table $\mathbb{Q}^{\mathrm{B} 5}$.

Indeed, as discussed in Section III the corresponding transmission in the HOMO-LUMO region of those junctions systematically drops down with the distance between the electrodes, as seen in Figs 3 and $[4$ The true discrete nature of molecular resonances starts to govern the transport. The level spacing of the resonances is illustrated in terms of the eigenvalues of the projected self-consistent Hamiltonian onto the molecular orbitals (MPSH) in Fig 8 The levels are more closely spaced and the broadening of the levels goes down with the length of the molecule.

The semiconducting property with a narrow band gap of the $\pi$ conjugated system is well known. The gap is due to a distinct alternation of the bonds, with every second bond having double-bond character. In the poly p-phenylene vinylene molecule, the HOMO-LUMO gap of $2.5 \mathrm{eV}$ has been reported ${ }^{36}$ The reduction of the gap obtained in this paper, as seen in Fig 8 is expected for the thiolate-ended molecule on gold surfaces.

Another characteristic feature of the OPVn series is its zero bias conductance. One finds a remarkable difference between the DTB and OPV5 systems. For the DTB, it is $35 \mu S$, while for the OPV5 of the same contact geometry it is only $0.2 \mu \mathrm{S}$. Obviously, the transmission in the HOMO-LUMO region appreciably drops down in the series as seen from Fig [5.7 For an even longer molecule, one expects a negligible density of states around $E_{f}$ and a vanishing transmission in the HOMO-LUMO region for low bias voltages.

These two features, the fixed HOMO-LUMO separation and the gap in the density of states around $E_{f}$, show that a kind of bias threshold sets up. A current through

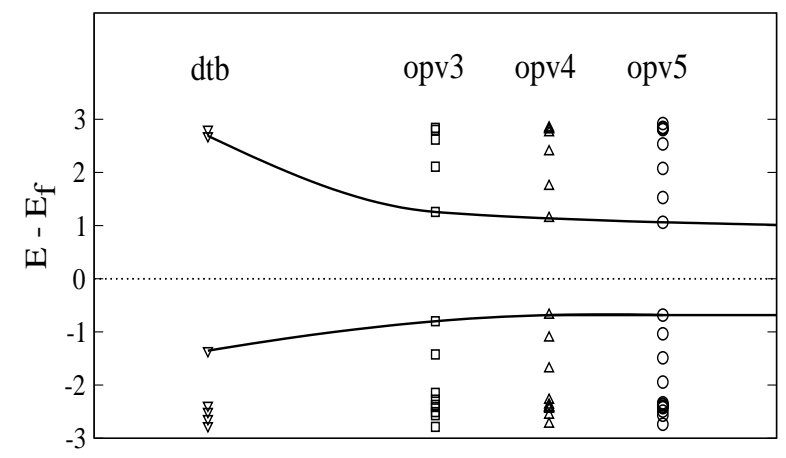

FIG. 8: Eigenvalues of the projected self-consistent Hamiltonian of the $\mathrm{Au}(111)$-molecule- $\mathrm{Au}(111)$ system onto the molecular orbitals at zero-bias. Full lines connect the HOMO and LUMO levels, respectively.

the junction will start at a bias above some threshold value, at which the wings of the HOMO and LUMO peaks enter the bias window. This is a kind of bias-activated coherent transport.

One expects the gap in the I-V curve to be more pronounced in the systems with weaker chemisorptions than in the sulfur-ended molecule on gold electrodes.

Owing to the bias gap, a long molecule, even though chemisorbed on the electrodes, may show a behaviour

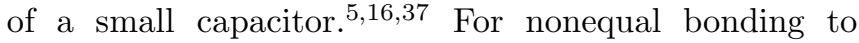
the electrodes, this quite often happens in real junctions. This opens the possibility for a kind of sequential transport in $\pi$ conjugated systems and charging of the strongly chemisorbed molecule in gate-controlled experiments. Kubatkin et al $\underline{\underline{9}}$ have demonstrated gatecontrolled Coulomb blockade involving different charge states in the OPV5 molecule of tran conformation, but with the protected thiol end-groups of their molecule, thus preventing strong chemisorption.

In the view of this, a long molecule coupled to the electrodes may also serve as a testing ground for the boundary region between mesoscopic and nanostructure systems.

We also point out that the calculations we have performed showed that the hollow position is the minimumenergy absorption site for all molecules in the series. We have taken the distance of sulfur to gold atoms as $5 \%$ larger and found that it does not change the conduction noticeably 38 Besides the known mechanical and temperature stability of thiolate-ended organic molecules bonded to gold surfaces, $\stackrel{5.6}{,}$ this robustness of conduction is an additional desirable property in the view of applicability in molecular electronics.

\section{CONCLUDING REMARKS}

Using the NEGF and DFT we have obtained the nonlinear current-voltage characteristics of thiolate-ended phenylene vinylene oligomers coupled to $\mathrm{Au}(111)$ elec- 
trodes. In the low-bias region we have found that the current scales down inversely to the interelectrode separation, with the strong tendency of the appearance of the I-V gap for long molecules. The reason for the gap formation is the narrowing of the HOMO and LUMO related transmission peaks with the interelectrode separation. The nonexistence of the I-V gap in the junctions of short molecules appears to be a consequence of the ideal $\mathrm{Au}(111)$ semi-infinite surfaces and the moleculemediated gap states encountered in the calculations resulting in wide resonances and finite density of states around the Fermi level. The measured conductance of short molecules is usually smaller than the calculated one, which is possibly due to such ideal conditions seldom, if ever, encountered in real junctions. For longer molecule, the effects appear to be less efficient, and as expected the discrepancies between the measured and cal- culated values are less pronounced. We point out that the full account of such effects could only be followed with the full ab initio self-consistent calculations we performed at each bias voltage.

We have also found that the conductance is robust to the sulfur gold distance for the molecules chemisorbed in the local energy minimum, hollow site on gold surfaces.

\section{Acknowledgments}

This work was supported in part by the Ministry of Science and Technology of the Republic of Croatia under contract No. 0098001, by the IST-FET-NANOMOL project of the EC, by the Swedish Research Council and by the Swedish Foundation for Strategic Research.
* crljen@irb.hr

1 A. Aviram, and M.A. Ratner, Chem. Phys. Lett. 29, 277 (1974).

2 J. Chen, M.A. Reed, A.M. Rawlett, J.M. Tour, Science 286, 1550 (1999).

3 C.P. Collier, G. Mattersteig, E.W. Wong, Y. Luo, K. Beverly, J. Sampaio, F.M. Raymo, J.F. Stoddart, and J.R. Heath, science 2891172 (2000).

4 J. Chen, and M.A. Reed, Chem. Phys. 281, 127 (2002).

${ }^{5}$ C. Kergueris, J-P. Bourgoin, S. Palacin, D. Esteve, C. Urbina, M. Magoga, and C. Joachim, Phys. Rev. B 59, 12505 (1999).

6 M.A. Reed, C. Zhou, C.J. Muller, T.P. Burgin, and J.M. Tour, Science 278, 252 (1997).

7 A. Aviram, C. Joachim, and M. Pomerantz, Chem. Phys. Lett. 146, 490 (1988).

8 J. Reichert, R. Ochs, D. Beckmann, H.B.Weber, M. Mayor, and H. v. Lóhneysen, Phys. Rev. Lett. 88, 176804 (2002).

9 S.E. Kubatkin, A. Danilov, M. Hjort, J. Cornil, J.L.Bredas, N. Stuhr-Hansen and T. Björnholm, Nature 425, 698 (2003).

10 N. B. Zhitenev, A. Erbe, and Z. Bao, Phys. Rev. Lett. 92, 186805 (2004).

11 S.N. Yaliraki, A.E.Roitberg, C. Gonzales, V. Mujica, and M.A. Ratner, J. Phys. Chem. 111, 6997 (1999).

12 P.A. Derosa, J.M. Seminario, J. Phys. Chem. B 105, 471 (2001).

13 Y. Xue, S. Datta, and M.A. Ratner, J. Chem. Phys. 115, 4292 (2001).

14 M. Di Ventra, S.T. Pantelides, and N.D. Lang, Phys. Rev. Lett. 84, 979 (2000).

15 K. Stokbro, J. Taylor, M. Brandbyge, J.-L. Mozos, and P. Ordejón, Comp. Mat. Sci., 27 151, (2003).

16 Mujica, J. Chem. Phys. 104, 7296 (1996).

17 J. Heurich, J.C. Cuevas, W. Wenzel, and G. Schön, Phys. Rev. Lett. 88, 256803 (2002).

18 J. Taylor, M. Brandbyge and K. Stokbro, Phys. Rev. B 68, 121101 (2003).

19 X.D. Cui, A. Primak, X. Zarate, J. Tomfohr, O.F. Sankey, A.L. Moore, T.A. Moore, D. Gust, G. Harris, and S.M. Lindsay, Science 294, 571 (2001).
20 J.M. Tour, Chem. Rev. 96, 537 (1996).

21 J. Roncali, Chem. Rev. 97, 173 (1997).

22 M. Magoga and C. Joachim, Phys. Rev. B 56, 4722 (1997), ibid. Phys. Rev. B 57, 1820 (1998).

23 Ž. Crljen, A. Grigoriev, G. Wendin, and K. Stokbro, oral presentation, EURESCO conference, Fundamental Aspects of Surface Science, Kerkrade, Netherland 2003.

24 A.Grigoriev, J. Skolberg, G. Wendin, Ž. Crljen, and K. Stokbro, to be published.

25 M. Brandbyge, J.L. Mozos, P. Ordejon, J. Taylor, K. Stokbro, Phys. Rev. B 65, 165401, (2002).

26 N. Troullier, J.L. Martins, Phys. Rev. B 43, 1993, (1991).

27 A double $\zeta+$ polarization basis for the OPVn molecule, while a double $\zeta$ for the gold $s$ channel and single $\zeta$ for the gold $p$ and $d$ channel was used.

28 Y. Xue, S. Datta, S. Hong, R. Reifenberger, J.I. Henderson and C.P. Kubiak, Phys. Rev. B 59, R7852 (1999).

29 The STM measurement of A. Szuchmacher Blum et al ${ }^{30}$ have shown that the isolated oligo phenylene ethynylene molecules have the same measured conductance as molecules in a crystalline self-assembled monolayer indicating that the transport occurs through the individual molecule with no effects of the neighbour molecules or intermolecular hopping.

30 A. Szuchmacher Blum, J.C.Yang, R. Shashidhar, and B. Ratna, Appl. Phys. Lett. 82, 3322 (2003).

31 K. Walzer, E. Marx, N.C.Greenham, R.J. Less, P.R. Raithby, and K. Stokbro, J. Am. Phys. Soc. 126, 1229 (2004)

32 W. Andreoni, A. Curioni, H. Grönbeck, Int. J. Quant. Chem. 80, 598 (2000).

33 E.G.Emberly and G. Kirczenow, Phys. Rev. B 58, 10911 (1998), J. Taylor, Ph.D. thesis, McGill University (2000).

34 A.W. Ghosh, F. Zahid, S. Datta, and R.R. Birge, Chem. Phys. 281, 225 (2002).

35 Our calculations do not show an exponential decrease of the conductance with the interelectrode separation for the lengths we have considered. That is a new feature with respect to the the semi-empirical approach of Ref. 22, showing that the full ab initio approach should be used for the junctions with chemisorbed molecules. 
${ }^{36}$ R.H. Friend, R.W. Gymer, A.B. Holmes, J.H. Burroughes, R.N. Marks, C. Taliani, D.D.C. Bradley, D.A. Dos Santos, J.L. Brédas, M. Lögdlund, and W.R. Salaneck, Nature 397, 121 (1999).

37 C. Zhou, M.R. Deshpande, M.A. Reed, L. Jones II, and J.M. Tour, Appl. Phys. Lett. 71, 611 (1997).

38 The change of the conductance with preasure in the AFM measurement might be due to some other transport mechanism or due to the change in the geometry of the system, like bending of the molecules, see C. Joachim, J.K. Gimzewski, R.R. Schlittler, and C. Chavy, Phys. Rev. Lett. 74, 2102 (1995); Leatherman, J. Phys.Chem. B 103, 4006 (1999). 